\title{
EXACTNESS OF ROKHLIN ENDOMORPHISMS AND WEAK MIXING OF POISSON BOUNDARIES.
}

\author{
Jon Aaronson \& Mariusz LemańCZyK
}

\begin{abstract}
We give conditions for the exactness of Rokhlin skew products, apply these to random walks on locally compact, second countable topological groups and obtain that the Poisson boundary of a globally supported random walk on such a group is weakly mixing.
\end{abstract}

\section{$\S 0$ INTRODUCTION}

\section{Skew product endomorphisms.}

Let $(X, \mathcal{B}, m, T)$ be a non-singular, exact endomorphism. Let $\mathbb{G}$ be a locally compact, Polish topological (LCP) group, let $f: X \rightarrow \mathbb{G}$ be measurable and let $S: \mathbb{G} \rightarrow \operatorname{Aut}(Y)$ be an ergodic, nonsingular $\mathbb{G}$-action on the probability space $(Y, \mathcal{C}, \nu)$ (i.e. $S$ is a measurable homomorphism). We give (theorem 2.3) conditions for the exactness of the Rokhlin endomorphism $\widetilde{T}=\widetilde{T}_{f, S}: X \times Y \rightarrow X \times Y$ defined by

$$
\widetilde{T}(x, y):=\left(T x, S_{f(x)} y\right) .
$$

These conditions are applied to random walk endomorphisms. Meilijson (in [Me]) gave sufficient conditions for exactness for random walk endomorphisms over $\mathbb{G}=\mathbb{Z}$. We clarify Meilijson's theorem, proving a converse (proposition 4.2), extend it to countable Abelian groups (theorem 4.1), characterize the exactness of the Rokhlin endomorphism for a steady random walk (theorem 4.5) and obtain that the right action on Poisson boundary (see $\S 4$ ) of an adapted (i.e. globally supported) random walk is weakly mixing (proposition 4.4). Tools employed include the ergodic theory of "associated actions" (see §1), and the boundary theory of random walks (see $\S 4$ ).

\section{$\S 1$ Associated actions}

For an endomorphism $R$ of a measure space $(Z, \mathcal{D}, \nu)$ set

- $\mathcal{I}(R):=\left\{A \in \mathcal{D}: R^{-1} A=A\right\}$ - the invariant $\sigma$-algebra, and

- $\mathcal{T}(R):=\bigcap_{n=0}^{\infty} R^{-n} \mathcal{D}$ - the tail $\sigma$-algebra.

Let $(X, \mathcal{B}, m, T)$ be a non-singular, exact endomorphism. Let $\mathbb{G}$ be a locally compact, Polish topological (LCP) group, let $f: X \rightarrow \mathbb{G}$ be measurable.

1991 Mathematics Subject Classification. 37A20, 37A15 (37A40, 60B15, 60J50).

(C)2004. Aaronson was partially supported by the EC FP5, IMPAN-BC Centre of Excellence when this work was begun. Lemańczyk's research was partially supported by KBN grant 1 P03A 03826. Both authors thank MPIM, Bonn for hospitality when this work was finished. 
There are two associated (right) $\mathbb{G}$-actions arising from the invariant and tail $\sigma$-algebras of $T_{f}$, which are defined as follows:

- define the (left) skew product endomorphism $T_{f}: X \times \mathbb{G} \rightarrow X \times \mathbb{G}$ by $T_{f}(x, g):=$ $(T x, f(x) g)$ and fix $\mathbb{P} \in \mathcal{P}(X \times \mathbb{G}), \mathbb{P} \sim m \times m_{\mathbb{G}} ;$

- for $t \in \mathbb{G}$, define $Q_{t}: X \times \mathbb{G} \rightarrow X \times \mathbb{G}$ by $Q_{t}(x, g):=(x, g t)$, then $Q_{t} \circ T_{f}=T_{f} \circ Q_{t}$.

\section{The associated invariant action.}

Let $(\Omega, \mathcal{F}, P)=\left(\Omega_{\mathcal{I}}, \mathcal{F}_{\mathcal{I}}, P_{\mathcal{I}}\right)$ be the invariant factor of $\left(X \times \mathbb{G}, \mathcal{B}(X \times \mathbb{G}), \mathbb{P}, T_{f}\right)$ : that standard probability space equipped with a measurable map $\pi: X \times \mathbb{G} \rightarrow \Omega$ such that $\mathbb{P} \circ \pi^{-1}=P, \pi \circ T_{f}=\pi$ and $\pi^{-1} \mathcal{F}=\mathcal{I}\left(T_{f}\right)$. Since $Q_{t} \mathcal{I}\left(T_{f}\right)=\mathcal{I}\left(T_{f}\right)$ (because $T_{f} \circ Q_{t}=Q_{t} \circ T_{f}$ ),

- $\exists$ a $P$-non-singular $\mathbb{G}$-action $\mathfrak{p}: \Omega \rightarrow \Omega$ so that $\pi \circ Q=\mathfrak{p} \circ \pi$.

Proposition 1.1. $(\Omega, \mathcal{F}, P, \mathfrak{p})$ is ergodic iff $(X, \mathcal{B}, m, T)$ is ergodic.

Proof. If $(X, \mathcal{B}, m, T)$ is ergodic, then so is $\left(X \times \mathbb{G}, \mathcal{B}(X \times \mathbb{G}), \mathbb{P},\left\langle T_{f}, Q\right\rangle\right)$ where $\left\langle T_{f}, Q\right\rangle$ denotes the $\mathbb{Z}_{+} \times \mathbb{G}$ action defined by $(n, t) \mapsto T_{f}^{n} \circ Q_{t} \in \operatorname{Aut}(X \times \mathbb{G})$. Any $\mathfrak{p}$-invariant, measurable function on $\Omega$ lifts by $\pi$ to a $\left\langle T_{f}, Q\right\rangle$-invariant, measurable function on $X \times \mathbb{G}$, which is constant $\mathbb{P}$-a.e..

Conversely, any $T$-invariant function on $X$ lifts to a $T_{f}$-invariant function on $X \times \mathbb{G}$ which is also $Q$-invariant and thus the lift of a $\mathfrak{p}$-invariant, measurable function on $\Omega$. If $(\Omega, \mathcal{F}, P, g)$ is ergodic this function is constant (a.e.).

The non-singular $\mathbb{G}$-action $(\Omega, \mathcal{F}, P, \mathfrak{p})$ is called the invariant $\mathbb{G}$-action associated to $(T, f)$ and denoted $\mathfrak{p}=\mathfrak{p}(T, f)$.

This action is related to the Mackey range of a cocycle (see [Zi2] and $\S 3$ ), and the Poisson boundary of a random walk (see $\S 4$ ).

\section{The associated tail action.}

Let $(\Omega, \mathcal{F}, P)=\left(\Omega_{\mathcal{T}}, \mathcal{F}_{\mathcal{T}}, P_{\mathcal{T}}\right)$ be the tail factor of $\left(X \times \mathbb{G}, \mathcal{B}(X \times \mathbb{G}), \mathbb{P}, T_{f}\right)$ : that standard probability space equipped with a measurable map $\pi: X \times \mathbb{G} \rightarrow \Omega$ such that $\mathbb{P} \circ \pi^{-1}=P, \pi^{-1} \mathcal{F}=\mathcal{T}\left(T_{f}\right)$. Since $Q_{t} \mathcal{T}\left(T_{f}\right)=\mathcal{T}\left(T_{f}\right)$ (because $T_{f} \circ Q_{t}=$ $\left.Q_{t} \circ T_{f}\right)$,

- $\exists$ a $P$-non-singular $\mathbb{G}$-action $\tau: \Omega \rightarrow \Omega$ so that $\pi \circ Q=\tau \circ \pi$.

Proposition 1.2. $(\Omega, \mathcal{F}, P, \tau)$ is ergodic iff $(X, \mathcal{B}, m, T)$ is exact.

Proof. Suppose first that $(X, \mathcal{B}, m, T)$ is exact. Any $\tau$-invariant, measurable function $F: \Omega \rightarrow \mathbb{R}$ lifts by $\pi$ to a $Q$-invariant, $\mathcal{T}\left(T_{f}\right)$-measurable function $\bar{F}: X \times \mathbb{G} \rightarrow$ $\mathbb{R}$. In particular $\exists \bar{F}_{n}: X \times \mathbb{G} \rightarrow \mathbb{R} \quad(n \geq 0)$, measurable so that $\bar{F}=\bar{F}_{n} \circ T_{f}^{n}$. It follows from $Q_{t} \circ T_{f}=T_{f} \circ Q_{t}$ that each $\bar{F}_{n}$ is $Q$-invariant, whence $\exists \hat{F}_{n}: X \rightarrow \mathbb{R}$ measurable so that $\bar{F}_{n}(x, y)=\hat{F}_{n}(x)$ for $\mathbb{P}$-a.e. $(x, y) \in X \times \mathbb{G}$. Thus $\bar{F}=\bar{F}_{0}=\hat{F}_{0}$ is $\mathcal{T}(T)$-measurable, whence constant $\mathbb{P}$-a.e. by exactness of $T$.

Conversely, any $\mathcal{T}(T)$-measurable function on $X$ lifts to a $\mathcal{T}\left(T_{f}\right)$-measurable function on $X \times \mathbb{G}$ which is also $Q$-invariant and thus the lift of a $\tau$-invariant, measurable function on $\Omega$. If $(\Omega, \mathcal{F}, P, \tau)$ is ergodic this function is constant (a.e.).

The non-singular $\mathbb{G}$-action $(\Omega, \mathcal{F}, P, \tau)$ is called the associated tail $\mathbb{G}$-action (of $(T, f))$ and denoted $\tau=\tau(T, f)$. This action is related to the tail boundary of a 


\section{$\S 2$ Conditions for exactness and a COnstruction of Zimmer}

We begin with a proposition generalising Zimmer's construction (in [Zi1]) of a $\mathbb{G}$-valued cocycle over an ergodic, probability preserving transformation with a prescribed ergodic, probability preserving $\mathbb{G}$-action as Mackey range.

\section{Proposition 2.1.}

Suppose that $\mathbb{G}$ is a LCP group, that $(X, \mathcal{B}, m, T)$ is a non-singular endomorphism, and suppose that $f: X \rightarrow \mathbb{G}$ is measurable.

Let $S$ be a non-singular $\mathbb{G}$-action on the probability space $(Y, \mathcal{C}, \nu)$ and define $\widetilde{T}=\widetilde{T}_{f, S}: X \times Y \rightarrow X \times Y$ by

$$
\widetilde{T}(x, y):=\left(T x, S_{f(x)} y\right)
$$

then

$$
\mathfrak{p}(\widetilde{T}, f) \cong \mathfrak{p}(T, f) \times S, \& \tau(\widetilde{T}, f) \cong \tau(T, f) \times S
$$

Proof.

Define $\pi: X \times Y \times \mathbb{G} \rightarrow X \times \mathbb{G} \times Y$ by $\pi(x, y, g):=\left(x, g, S_{g^{-1}} y\right)$. Evidently $\pi$ is a bimeasurable bijection.

Fixing $p \in \mathcal{P}(\mathbb{G}), p \sim m_{\mathbb{G}}$, we claim that

$$
(m \times \nu \times p) \circ \pi^{-1} \sim m \times p \times \nu
$$

moreover

$$
\frac{d(m \times \nu \times p) \circ \pi^{-1}}{d(m \times p \times \nu)}(x, g, y)=\frac{d \nu \circ S_{g}}{d \nu}(y)=: D(g, y)
$$

To see this, let $a \in L^{1}(X, m), b \in L^{1}(Y, \nu)$ and $c \in L^{1}(\mathbb{G}, p)$, then

$$
\begin{aligned}
\int_{X \times \mathbb{G} \times Y}(a \otimes b \otimes c) \circ \pi \quad & d(m \times \nu \times p)=\int_{X} a d m \int_{\mathbb{G}} c(g)\left(\int_{Y} b \circ S_{g}^{-1} d \nu\right) d p(g) \\
& =\int_{X} a d m \int_{\mathbb{G}} c(g)\left(\int_{Y} b \frac{d \nu \circ S_{g}}{d \nu} d \nu\right) d p(g) \\
& =\int_{X} a d m \int_{\mathbb{G} \times Y} b(y) c(g) \frac{d \nu \circ S_{g}}{d \nu}(y) d(p \times \nu)(g, y) \\
& =\int_{X \times \mathbb{G} \times Y} a \otimes b \otimes c \otimes g D d(m \times p \times \nu) .
\end{aligned}
$$

Next, we claim that

$$
\pi \circ \widetilde{T}_{f}=\left(T_{f} \times\left.\mathrm{Id}\right|_{Y}\right) \circ \pi
$$

To see this,

$$
\pi \circ \widetilde{T}_{f}(x, y, g)=\pi\left(T x, S_{f(x)} y, f(x) g\right)=\left(T x, f(x) g, S_{(f(x) g)^{-1}} S_{f(x)} y\right)
$$


It follows from (2) that

$$
\mathcal{I}\left(\widetilde{T}_{f}\right)=\pi^{-1}\left(\mathcal{I}\left(T_{f} \times \text { Id }\right)\right), \quad \mathcal{T}\left(\widetilde{T}_{f}\right)=\pi^{-1}\left(\mathcal{T}\left(T_{f} \times \text { Id }\right)\right) .
$$

Now, in general

$$
\mathcal{I}\left(T_{f} \times \mathrm{Id}\right)=\mathcal{I}\left(T_{f}\right) \otimes \mathcal{B}(Y), \quad \mathcal{T}\left(T_{f} \times \mathrm{Id}\right)=\mathcal{T}\left(T_{f}\right) \otimes \mathcal{B}(Y) \quad \bmod \quad m \times p \times \nu
$$

and so

$$
\mathcal{I}\left(\widetilde{T}_{f}\right)=\pi^{-1}\left(\mathcal{I}\left(T_{f}\right) \otimes \mathcal{B}(Y)\right), \quad \mathcal{T}\left(\widetilde{T}_{f}\right)=\pi^{-1}\left(\mathcal{T}\left(T_{f}\right) \otimes \mathcal{B}(Y)\right) \quad \bmod \quad m \times p \times \nu
$$

Now let $\left(\Omega_{i}, \mathcal{F}_{i}, P_{i}\right)$ and let $\left(\widetilde{\Omega}_{i}, \widetilde{\mathcal{F}}_{i}, \widetilde{P}_{i}\right) \quad(i=\mathfrak{p}, \tau)$ be the invariant or tail factors of $\left(X \times \mathbb{G}, m \times p, T_{f}\right)$ and $\left(X \times Y \times \mathbb{G}, m \times \nu \times p, \widetilde{T}_{f}\right)$ respectively, according to the value of $i=\mathfrak{p}, \tau$. By (1) and (2), $\pi$ induces a measure space isomorphism of $\left(\widetilde{\Omega}_{i}, \widetilde{P}_{i}\right)$ with $\left(\Omega_{i} \times Y, P_{i} \times \nu\right)$.

Denoting the associated $\mathbb{G}$-actions by $\widetilde{Q}_{t}(x, y, g):=(x, y, g t)$ and $Q_{t}(x, g):=$ $(x, g t)$, we note that

$$
\pi \circ \widetilde{Q}_{t}^{-1}=\left(Q_{t}^{-1} \times S_{t}\right) \circ \pi
$$

The proposition now follows from this.

\section{Corollary 2.2.}

1) $\widetilde{T}$ is ergodic iff $\mathfrak{p}(T, f) \times S$ is ergodic.

2) $\widetilde{T}$ is exact iff $\tau(T, f) \times S$ is ergodic.

3) If both $T_{f}$ and $S$ are ergodic, then $\widetilde{T}$ is ergodic and $\mathfrak{p}(\widetilde{T}, f) \cong S$.

4) If $T_{f}$ is exact and $S$ is ergodic, then $\widetilde{T}$ is exact and $\tau(\widetilde{T}, f) \cong S$.

Proof. Parts 1) and 2) follow from propositions 1.1 and 1.2. Parts 3) and 4) follow from these and form essentially Zimmer's construction.

\section{$\S 3$ LOCALLY INVERTIBLE ENDOMORPHISMS}

In this section, we obtain additional results for a non-singular, exact endomorphism $(X, \mathcal{B}, m, T)$ of a standard measure space which is locally invertible in the sense that $\exists$ an at most countable partition $\alpha \subset \mathcal{B}$ so that $T: a \rightarrow T a$ is invertible, non-singular. Under the assumption of local invertibility, the associated actions of $\S 1$ are Mackey ranges of cocycles (as in [Zi2]). We prove

\section{Theorem 3.1.}

Suppose that $(X, \mathcal{B}, m, T)$ is a non-singular, locally invertible, exact endomorphism of a standard measure space, that $\mathbb{G}$ is locally compact and Abelian and that $f: X \rightarrow \mathbb{G}$ is measurable.

Either $\widetilde{T}_{f, S}$ is exact for every mildly mixing probability preserving $\mathbb{G}$-action $S$ : $\mathbb{G} \rightarrow \operatorname{Aut}(Y)$, or $\exists$ a compact subgroup $\mathbb{K} \leq \mathbb{G}, t \in \mathbb{G}$ and $\bar{f}: X \rightarrow \mathbb{K}, g: X \rightarrow \mathbb{G}$ measurable so that

$$
f=g-g \circ T+t+\bar{f}
$$

Note that the invertible version of this generalizes corollary 6 of $[\mathrm{Ru}]$. The rest 


\section{Tail relations.}

Let $(X, \mathcal{B}, m, T)$ be a non-singular, locally invertible endomorphism of a standard measure space. Consider the tail relations

$$
\begin{gathered}
\mathfrak{T}(T):=\left\{(x, y) \in X \times X: \exists k \geq 0, T^{k} x=T^{k} y\right\} ; \\
\mathfrak{G}(T):=\left\{(x, y) \in X_{0} \times X_{0}: \exists k, \ell \geq 0, T^{k} x=T^{\ell} y\right\}
\end{gathered}
$$

where $X_{0}:=\left\{x \in X: T^{n+k} x \neq T^{k} x \forall n, k \geq 1\right\}$. We assume that $m\left(X \backslash X_{0}\right)=0$ (which is the case if $T$ is ergodic and $m$ is non-atomic) and so $\mathfrak{T}(T) \subset \mathfrak{G}(T)$ $\bmod m$. Both $\mathfrak{T}(T)$ and $\mathfrak{G}(T)$ are standard, countable, $m$-non-singular equivalence relations in sense of $[\mathrm{F}-\mathrm{M}]$ whose invariant sets are given by

$$
\mathcal{I}(\mathfrak{G}(T))=\mathcal{I}(T), \quad \mathcal{I}(\mathfrak{T}(T))=\mathcal{T}(T)
$$

respectively.

Given a LCP group $\mathbb{G}$ and $f: X \rightarrow \mathbb{G}$ measurable, define $f_{n}: X \rightarrow \mathbb{G}(n \geq 1)$ by

$$
f_{n}(x):=f\left(T^{n-1} x\right) f\left(T^{n-2} x\right) \ldots f(T x) f(x)
$$

and define $\Psi_{f}: \mathfrak{G}(T) \rightarrow \mathbb{G}$ by

$$
\Psi_{f}\left(x, x^{\prime}\right):=f_{\ell}\left(x^{\prime}\right)^{-1} f_{k}(x) \text { for } k, \ell \geq 0 \text { such that } T^{k} x=T^{\ell} x^{\prime}
$$

(this does not depend on the $k, \ell \geq 0$ such that $T^{k} x=T^{\ell} x^{\prime}$ for $x, x^{\prime} \in X_{0}$ ).

It follows that $\Psi_{f}: \mathfrak{G}(T) \rightarrow \mathbb{G}$ is a (left) $\mathfrak{G}(T)$-orbit cocycle in the sense that

$$
\Psi_{f}(y, z) \Psi_{f}(x, y)=\Psi_{f}(x, z) \forall(x, y),(y, z) \in \mathfrak{G}(T) .
$$

Note that since $\mathfrak{T}(T) \subset \mathfrak{G}(T) \quad \bmod m$, the restriction $\Psi_{f}: \mathfrak{T}(T) \rightarrow \mathbb{G}$ is a (left) $\mathfrak{T}(T)$-orbit cocycle.

Mackey ranges of cocycles. Let $\mathcal{R}$ be a countable, standard, non-singular equivalence relation on the standard measure space $(X, \mathcal{B}, m)$ and let $\Psi: \mathcal{R} \rightarrow \mathbb{G}$ be a left $\mathcal{R}$-orbit cocycle. It follows from theorem 1 in $[\mathrm{F}-\mathrm{M}]$, there is a countable group $\Gamma$ and a non-singular $\Gamma$-action $(X, \mathcal{B}, m, V)$ so that

$$
\mathcal{R}=\mathcal{R}_{V}:=\left\{\left(x, V_{\gamma} x\right): \gamma \in \Gamma, x \in X\right\} .
$$

Let $f(\gamma, x):=\Psi\left(x, V_{\gamma} x\right) \quad\left(f=f_{\Psi, V}: \Gamma \times X \rightarrow \mathbb{G}\right)$ be the associated left $V$-cocycle (satisfying $\left.f\left(\gamma \gamma^{\prime}, z\right)=f\left(\gamma, V_{\gamma^{\prime}} z\right) f\left(\gamma^{\prime}, z\right)\right)$.

The Mackey range $\mathfrak{R}(V, f)$ (see [Zi2]) is analogous to the invariant action of $\S 1$. It is the non-singular $\mathbb{G}$-action of $Q \quad\left(Q_{t}(x, y):=(x, y t)\right)$ on the invariant factor of $V_{f}: X \times \mathbb{G} \rightarrow X \times \mathbb{G} \quad\left(\left(V_{f}\right)_{\gamma}(x, g):=\left(V_{\gamma} x, f(\gamma, x) g\right)\right)$.

As before, $\mathfrak{R}(V, f)$ is ergodic iff $(X, \mathcal{B}, m, V)$ is ergodic.

It can be shown that $\mathfrak{R}\left(V, f_{\Psi, V}\right)$ does not depend on $V$ such that $\mathcal{R}=\mathcal{R}_{V}$ and we define the Mackey range of $\Psi$ over $\mathcal{R}$ as $\mathfrak{R}(\mathcal{R}, \Psi):=\mathfrak{R}\left(V, f_{\Psi, V}\right)$.

\section{Proposition 3.2.}

$$
\mathfrak{p}(T, f)=\mathfrak{R}\left(\mathfrak{G}(T), \Psi_{f}\right), \quad \tau(T, f)=\mathfrak{R}\left(\mathfrak{T}(T), \Psi_{f}\right)
$$




\section{Proposition 3.3.}

Suppose that $\Gamma$ is a countable group, that $\mathbb{G}$ is a LCP group, that $(X, \mathcal{B}, m, V)$ is an ergodic, non-singular $\Gamma$-action, and suppose that $f: \Gamma \times X \rightarrow$ $\mathbb{G}$ is a measurable cocycle.

Let $S$ be a non-singular $\mathbb{G}$-action on the probability space $(Y, \mathcal{C}, \nu)$ and define $\widetilde{V}: \Gamma \rightarrow \operatorname{Aut}(X \times Y)$ by $\widetilde{V}_{\gamma}(x, y):=\left(V_{\gamma} x, S_{f(\gamma, x)}(y)\right) ;$ then

$$
\mathfrak{R}(\widetilde{V}, f) \cong \mathfrak{R}(V, f) \times S
$$

\section{Ergodicity of Cartesian products.}

Call a probability preserving $\mathbb{G}$-action $(Y, \mathcal{C}, \nu, S)$ mildly mixing if

$$
f \in L^{2}(\nu), g_{n} \in \mathbb{G}, g_{n} \rightarrow \infty, f \circ S_{g_{n}} \stackrel{L^{2}(\nu)}{\rightarrow} f \Rightarrow f \text { is constant. }
$$

As shown in $[\mathrm{S}-\mathrm{W}]$, if the ergodic, nonsingular $\mathbb{G}$-action $(X, \mathcal{B}, m, U)$ is properly ergodic in the sense that $m\left(U_{\mathbb{G}}(x)\right)=0 \forall x \in X$, then $U \times S$ is ergodic for any mildly mixing probability preserving $\mathbb{G}$-action $S: \mathbb{G} \rightarrow \operatorname{Aut}(Y)$. The same proof shows that this result persists for an ergodic, nonsingular $\mathbb{G}$-action $(X, \mathcal{B}, m, U)$ which has a recurrent sequence, i.e. a sequence $g_{n} \in \mathbb{G}, g_{n} \rightarrow \infty$ with the property that

$$
\liminf _{n \rightarrow \infty}\left|f \circ U_{g_{n}}-f\right|=0 \quad m-\text { a.e. } \forall f: X \rightarrow \mathbb{R} \text { measurable. }
$$

\section{Compact reducibility.}

Let $\Gamma$ be a countable group, $\mathbb{G}$ be a LCP group and let $(X, \mathcal{B}, m, V)$ be an ergodic, non-singular $\Gamma$-action.

We call the measurable $V$-cocycle $F: \Gamma \times X \rightarrow \mathbb{G}$ compactly reducible if $\exists \mathbb{H} \leq \mathbb{G}$ compact, a measurable cocycle $f: \Gamma \times X \rightarrow \mathbb{H}$ and $h: X \rightarrow \mathbb{G}$ measurable so that $F\left(V_{\gamma}, x\right)=h\left(V_{\gamma} x\right)^{-1} f\left(V_{\gamma}, x\right) h(x)$.

Remark. Note that if $F$ is compactly reducible, then (by possibly reducing to a further subgroup) it can be ensured that $\left(X \times \mathbb{H}, \mathcal{B}(X \times \mathbb{H}), m \times m_{\mathbb{H}}, V_{f}\right)$ is ergodic (see $[\mathrm{Zi} 2])$.

Proposition 3.4. If the measurable $V$-cocycle $F: \Gamma \times X \rightarrow \mathbb{G}$ is not compactly reducible, then $\mathfrak{R}(V, F) \times S$ is ergodic for any mildly mixing

probability preserving $\mathbb{G}$-action $S: \mathbb{G} \rightarrow \operatorname{Aut}(Y)$.

Proof. Suppose that the conclusion fails, then $\mathfrak{R}(V, F)$ is not properly ergodic. It follows from [Zi2], proposition 4.2.24 that $\exists \mathbb{H} \leq \mathbb{G}$, a measurable cocycle $f: \Gamma \times X \rightarrow \mathbb{H}$ and $h: X \rightarrow \mathbb{G}$ measurable so that $F\left(V_{\gamma}, x\right)=h\left(V_{\gamma} x\right)^{-1} f\left(V_{\gamma}, x\right) h(x)$ and such that $\left(X \times \mathbb{H}, \mathcal{B}(X \times \mathbb{H}), m \times m_{\mathbb{H}}, V_{f}\right)$ is ergodic. In this case, $\mathfrak{R}(V, F)$ is the right action of $\mathbb{G}$ on $\mathbb{H} \backslash \mathbb{G}$ and there is no recurrent sequence when and only when $\mathbb{H}$ is compact.

Regularity and range of an orbit cocycle. Let $\mathbb{G}$ be a LCP group and let $\mathcal{R} \in \mathcal{B}(X \times X)$ be a standard, countable, non-singular equivalence relation. We call the left $\mathcal{R}$-orbit cocycle $\Psi: \mathcal{R} \rightarrow \mathbb{G}$ compactly reducible if the associated $f=f_{\Psi, V}$ has this property for some (and hence every) non-singular $\Gamma$-action $(X, \mathcal{B}, m, V)$ with $\mathcal{R}=\mathcal{R}_{V}$. 


\section{Proposition 3.5.}

If $\Psi$ is not compactly reducible, then $\mathfrak{R}(\mathcal{R}, \Psi) \times S$ is ergodic for any mildly mixing probability preserving $\mathbb{G}$-action $S: \mathbb{G} \rightarrow \operatorname{Aut}(Y)$.

We now have by proposition 3.5 that

\section{Proposition 3.6.}

For a locally invertible, exact endomorphism $(X, \mathcal{B}, m, T)$ and $f: X \rightarrow \mathbb{G}$ measurable: If $\Psi_{f}$ is not $\mathfrak{T}(T)$-compactly reducible, then $\tau(T, f) \times S$ is ergodic for any mildly mixing

probability preserving $\mathbb{G}$-action $S: \mathbb{G} \rightarrow \operatorname{Aut}(Y)$.

\section{Proof of theorem 3.1.}

The previous propositions show that if $\Psi_{f}$ is not $\mathfrak{T}(T)$-compactly reducible, then $\widetilde{T}_{f, S}$ is exact for every mildly mixing probability preserving $\mathbb{G}$-action $S: \mathbb{G} \rightarrow \operatorname{Aut}(Y)$.

We must show that $\Psi_{f}$ is $\mathfrak{T}(T)$-compactly reducible iff $\exists$ a compact subgroup $\mathbb{K} \leq \mathbb{G}, t \in \mathbb{G}$ and $\bar{f}: X \rightarrow \mathbb{K}, g: X \rightarrow \mathbb{G}$ measurable so that

$$
f=g-g \circ T+t+\bar{f} .
$$

To see this suppose that $\mathbb{K} \leq \mathbb{G}$ is a compact subgroup and $g: X \rightarrow \mathbb{G}$ is measurable so that the quotient cocycle (under the map $s \mapsto \widetilde{s}:=s+\mathbb{K}(\mathbb{G} \rightarrow$ $\mathbb{G} / \mathbb{K}))$ is a coboundary, i.e. $\widetilde{\Psi}_{f}\left(x, x^{\prime}\right)=\widetilde{g}(x)-\widetilde{g}\left(x^{\prime}\right)$. Set $h(x):=\widetilde{f}(x)-\widetilde{g}(x)+$ $\widetilde{g}(T x)$.

We claim that $h$ is $\mathfrak{T}(T)$-invariant, whence constant.

To prove this $\mathfrak{T}(T)$-invariance, (as in proposition 1.2 of [ANS]), note that $T^{n} x=$ $T^{n} x^{\prime} \Rightarrow h_{n}(x)-h_{n}\left(x^{\prime}\right)=\widetilde{f}_{n}(x)-\widetilde{f}_{n}\left(x^{\prime}\right)-\widetilde{g}(x)+\widetilde{g}\left(x^{\prime}\right)=0$. Also $T^{n} x=$ $T^{n} x^{\prime} \Rightarrow T^{n-1}(T x)=T^{n-1}\left(T x^{\prime}\right) \Rightarrow h_{n-1}(T x)=h_{n-1}\left(T x^{\prime}\right)$. Since $h(x)=$ $h_{n}(x)-h_{n-1}(T x)$, we have $h(x)=h\left(x^{\prime}\right)$.

\section{$\S 4$ RANDOM WALKS}

Let $\mathbb{G}$ be a LCP group and let $p \in \mathcal{P}(\mathbb{G})$.

The (left) random walk on $\mathbb{G}$ with jump probability $p \in \mathcal{P}(\mathbb{G})(\mathrm{RW}(\mathbb{G}, p)$ for short) is the stationary, one-sided shift of the Markov chain on $\mathbb{G}$ with transition probability $P(g, A):=p\left(A g^{-1}\right) \quad(A \in \mathcal{B}(\mathbb{G}))$.

This is isomorphic to the measure preserving transformation

$$
\left(X \times \mathbb{G}, \mathcal{B}(X \times \mathbb{G}), \mu_{p} \times m_{\mathbb{G}}, W\right)
$$

where $X=\mathbb{G}^{\mathbb{N}}, \mu_{p}: \mathcal{B}(X) \rightarrow[0,1]$ is the product measure $\mu_{p}:=p^{\mathbb{N}}$ defined by $\mu_{p}\left(\left[A_{1}, \ldots, A_{n}\right]\right):=\prod_{k=1}^{n} p\left(A_{k}\right)$ where $\left[A_{1}, \ldots, A_{n}\right]:=\left\{x \in X: x_{k} \in A_{k} \forall 1 \leq\right.$ $k \leq n\},\left(n \geq 1, A_{1}, \ldots, A_{n} \in \mathcal{B}(\mathbb{G})\right), m_{\mathbb{G}}$ is left Haar measure on $\mathbb{G}$ and $W$ : $X \times \mathbb{G} \rightarrow X \times \mathbb{G}$ is defined by $W(x, g):=\left(T x, x_{1} g\right)$ with $T: X \rightarrow X$ being the shift $(T x)_{n}:=x_{n+1}$.

The jump process of the random walk $\mathrm{RW}(\mathbb{G}, p)$ is

$$
\left(X, \mathcal{B}(X), \mu_{p}, T, f\right)
$$




\section{Boundaries.}

The tail boundary of the random walk $\operatorname{RW}(\mathbb{G}, p)$ is $\tau(\mathbb{G}, p):=\tau(T, f)$, and the Poisson boundary is $\mathfrak{p}(\mathbb{G}, p):=\mathfrak{p}(T, f)$ where $\left(X, \mathcal{B}(X), \mu_{p}, T, f\right)$ is the jump process of the random walk $\mathrm{RW}(\mathbb{G}, p)$.

These definitions are equivalent with those in $[\mathrm{K}-\mathrm{V}, \mathrm{K}]$ (see also $[\mathrm{Fu}]$ ) .

Random walks on Abelian groups. In case $\mathbb{G}$ is Abelian, the tail and Poisson boundaries are the actions of $\mathbb{G}$ on $\mathbb{G} / \mathbb{H}_{\tau}$ and $\mathbb{G} / \mathbb{H}_{\mathfrak{p}}$ by translation (respectively), where

$$
\mathbb{H}_{\mathfrak{p}}:=\overline{\langle\operatorname{supp} p\rangle}, \mathbb{H}_{\tau}:=\overline{\langle\operatorname{supp} p-\operatorname{supp} p\rangle} \text {. }
$$

See $[\mathrm{D}-\mathrm{L}]$.

\section{Theorem 4.1 (extension of $[\mathrm{Me}]$ ).}

Let $\mathbb{G}$ be a LCP, Abelian group, let $p \in \mathcal{P}(\mathbb{G})$ and let $\left(X, \mathcal{B}(X), \mu_{p}, T, f\right)$ be the jump process of the random walk $\mathrm{RW}(\mathbb{G}, p)$.

1) If $\mathbb{G} / \mathbb{H}_{\mathfrak{p}}\left(\mathbb{G} / \mathbb{H}_{\tau}\right)$ is compact and $S: \mathbb{G} \rightarrow$ Aut $(Y)$ is a weakly mixing probability preserving $\mathbb{G}$-action, then $\widetilde{T}_{f, S}$ is ergodic (exact).

2) If $\mathbb{H}_{\mathfrak{p}}\left(\mathbb{H}_{\tau}\right)$ is non-compact and $S: \mathbb{G} \rightarrow$ Aut $(Y)$ is a mildly mixing probability preserving $\mathbb{G}$-action, then $\widetilde{T}_{f, S}$ is ergodic (exact).

Proof.

1) It follows from the assumption that $\mathfrak{p}(T, f)(\tau(T, f))$ is an ergodic, probability preserving $\mathbb{G}$-action, whence $S \times \mathfrak{p}(T, f)(S \times \tau(T, f))$ is ergodic whenever $S$ is weakly mixing.

2) It follows from the assumption that $\mathfrak{p}(T, f)(\tau(T, f))$ is a conservative, ergodic, nonsingular $\mathbb{G}$-action, whence $S \times \mathfrak{p}(T, f)(S \times \tau(T, f))$ is ergodic whenever $S$ is mildly mixing.

Remark. If $\mathbb{H}_{\mathfrak{p}}\left(\mathbb{H}_{\tau}\right)$ is compact and $S: \mathbb{G} \rightarrow \operatorname{Aut}(Y)$ is a non-trivial probability preserving $\mathbb{G}$-action, then $\widetilde{T}_{f, S}$ is not ergodic (exact) since $\mathfrak{p}(T, f) \quad(\tau(T, f))$ is dissipative, whence $S \times \mathfrak{p}(T, f)(S \times \tau(T, f))$ is not ergodic.

\section{Proposition 4.2 (converse of $[\mathrm{Me}]$ ).}

Let $p \in \mathcal{P}(\mathbb{Z})$ and let $\left(X, \mathcal{B}(X), \mu_{p}, T, f\right)$ be the jump process of the random walk $\mathrm{RW}(\mathbb{Z}, p)$. Suppose

$$
\mathbb{H}:=\overline{\langle\operatorname{supp}(p)-\operatorname{supp}(p)\rangle}=d \mathbb{Z}
$$

with $d \neq\{0\}$ and let $S$ be an ergodic, probability preserving transformation of $Y$, then $\widetilde{T}_{f, S}$ is exact iff $S^{d}$ is ergodic.

Proof. Here, $\tau(T, f)$ is the cyclic permutation of $d$ points. The condition $S^{d}$ is ergodic characterizes the ergodicity of $S \times \tau\left(\mathfrak{T}(T), \psi_{f}\right)$.

\section{Weak mixing of Poisson boundary.}

Let $\mathbb{G}$ be a LCP group and suppose that $p \in \mathcal{P}(\mathbb{G})$ is globally supported in the sense that $\overline{\langle\operatorname{supp}(p)\rangle}=\mathbb{G}$. Let $\left(X, \mathcal{B}(X), \mu_{p}, T, f\right)$ be the jump process of the random walk $\mathrm{RW}(\mathbb{G}, p)$.

\section{Proposition 4.3.}

Suppose that $S: \mathbb{G} \rightarrow \operatorname{Aut}(Y)$ is a probability preserving $\mathbb{G}$-action, then $\widetilde{T}_{f, S}$ is ergodic iff $S$ is ergodic. 
Suppose that $F: X \times Y \rightarrow \mathbb{R}$ is bounded, measurable and $\widetilde{T}_{f, S}$-invariant, then $\widehat{\widetilde{T}_{f, S}} F=F$ where $\widehat{\widetilde{T}_{f, S}}: L^{1}\left(\mu_{p} \times \mu\right) \rightarrow L^{1}\left(\mu_{p} \times \mu\right)$ is the predual of

$$
f \mapsto f \circ \widetilde{T}_{f, S}\left(L^{\infty}\left(\mu_{p} \times \mu\right) \rightarrow L^{\infty}\left(\mu_{p} \times \mu\right)\right) .
$$

As in [Mo] (or proposition 1 of [ADSZ]), $\exists G: Y \rightarrow \mathbb{R}$ measurable so that $F(x, y)=$ $G(y) \mu_{p} \times \mu$-a.e., whence $G \circ S_{g}=G \mu$-a.e. $\forall g \in\langle\operatorname{supp}(p)\rangle=\mathbb{G}$ and $G$ (whence $F)$ is a.e. constant by ergodicity of $S$.

\section{Proposition 4.4.}

$\mathfrak{p}(T, f)$ is weakly mixing in the sense that $\mathfrak{p}(T, f) \times S$ whenever $S$ is an ergodic, probability preserving $\mathbb{G}$-action.

Proof. Let $S: \mathbb{G} \rightarrow$ Aut $(Y)$ be an ergodic, probability preserving $\mathbb{G}$-action. By proposition $4.3, \widetilde{T}_{f, S}$ is ergodic, whence by theorem $2.4, \mathfrak{p}(T, f) \times S$ is ergodic.

\section{Remark.}

More generally, propositions 4.3 and 4.4 remain true whenever $(X, \mathcal{B}, m, T, \alpha)$ is a probability preserving, weak quasi-Markov, almost onto fibred system in the sense of [ADSZ] and $f: X \rightarrow \mathbb{G}$ is $\alpha$-measurable and $m \circ f^{-1}$ is globally supported on $\mathbb{G}$.

\section{Aperiodic random walks.}

Let $\mathbb{G}$ be a LCP group and suppose that $p \in \mathcal{P}(\mathbb{G})$.

A random walk $\mathrm{RW}(\mathbb{G}, p)$ is called steady (see $[\mathrm{K}])$ if $\mathfrak{p}(\mathbb{G}, p)=\tau(\mathbb{G}, p)$.

Let $\mathbb{G}$ be a countable group and suppose that $p \in \mathcal{P}(\mathbb{G})$. The random walk $\operatorname{RW}(\mathbb{G}, p)$ is called aperiodic if the corresponding Markov chain is aperiodic. Equivalent conditions for this are

- $p_{e}^{n *}>0 \forall n$ large;

- $\exists n \geq 1$ so that $\operatorname{supp}\left(p^{n *}\right) \cap \operatorname{supp}\left(p^{(n+1) *}\right) \neq \emptyset$.

An aperiodic random walk on a countable group is steady. This can be gleaned from $[\mathrm{Fo}]$ and théorème 3 in [D] (see also proposition 4.5 in $[\mathrm{K}]$ ).

\section{Theorem 4.5.}

Let $\mathbb{G}$ be a LCP group and suppose that $p \in \mathcal{P}(\mathbb{G})$ is globally supported and that $\operatorname{RW}(\mathbb{G}, p)$ is steady. Let $\left(X, \mathcal{B}(X), \mu_{p}, T, f\right)$ be the jump process of the random walk RW $(\mathbb{G}, p)$ and let $S: \mathbb{G} \rightarrow$ Aut $(Y)$ be a probability preserving $\mathbb{G}$-action, then $\widetilde{T}_{f, S}$ is ergodic iff $S$ is ergodic and in this case $\widetilde{T}_{f, S}$ is exact.

Proof of $S$ ergodic $\Rightarrow \widetilde{T}_{f, S}$ exact. By proposition $4.4, \mathfrak{p}(T, f) \times S$ is ergodic, whence by steadiness, $\tau(T, f) \times S$ is ergodic. Thus, $\widetilde{T}_{f, S}$ is exact by theorem 2.3 .

\section{REFERENCES}

[ADSZ] J. Aaronson, M. Denker, O. Sarig, R. Zweimüller, Aperiodicity of cocycles and conditional local limit theorems, Stochastics and Dynamics 4 (2004), No. 1, 31-62.

[ANS] J. Aaronson, H. Nakada, O. Sarig, Exchangeable measures for subshifts, http://arxiv.org/abs/math.DS/0406578.

[D] Y. Derriennic, Lois "zéro ou deux" pour les processus de Markov. Applications aux marches alé atoires, Ann. Inst. H. Poincaré Sect. B (N.S.) 12 (1976), no. 2, 111-129.

[D-L] Y. Derriennic, M. Lin, Sur la tribu asymptotique des marches aléatoires sur les 
[F-M] J.Feldman, C.C.Moore, Ergodic equivalence relations, cohomology, and von Neumann algebras.I, Trans. Am. Math. Soc. 234 (1977), 289-324.

[Fo] S. Foguel, Iterates of a convolution on a non-abelian group, Ann. Inst. H. Poincar'e, Sect. B 11 (1975), 199-202.

[Fu] H. Furstenberg, Boundary theory and stochastic processes on homogeneous spaces, Harmonic analysis on homogeneous spaces, Proc. Sympos. Pure Math., Vol. XXVI, Williams Coll., Williamstown, Mass., 1972, Amer. Math. Soc., Providence, R.I., 1973, pp. 193-229.

[K] V.A. Kaimanovich, Measure-theoretic boundaries of Markov chains, 0-2 laws and entropy, Harmonic analysis and discrete potential theory (Frascati, 1991), Plenum, New York, 1992, pp. 145-180.

[K-V] V.A. Kaimanovich, A.M. Vershik, Random walks on discrete groups: boundary and entropy, Ann. Probab. 11 (1983), no. 3, 457-490.

[Me] I.Meilijson, Mixing properties of a class of skew-products, Israel J. Math. 19 (1974), 266-270.

[Mo] T. Morita, Deterministic version lemmas in ergodic theory of random dynamical systems, Hiroshima Math. J. 18 (1988), no. 1, 15-29.

[Ru] D.J. Rudolph, $Z^{n}$ and $R^{n}$ cocycle extensions and complementary algebras, Ergodic Theory Dynam. Systems 6 (1986), no. 4, 583-599.

[S-W] K. Schmidt, P. Walters, Mildly mixing actions of locally compact groups, Proc. Lon. Math. Soc. 45 (1982), no. 3, 506-518.

[Zi1] R.J. Zimmer, Amenable ergodic group actions and an application to Poisson boundaries of random walks, J. Functional Analysis 27 (1978), no. 3, 350-372.

[Zi2] _ Ergodic theory and semisimple groups, Monographs in Mathematics, vol 81, Birkhäuser Verlag, Basel, 1984.

[Aaronson] School of Math. Sciences, Tel Aviv University, 69978 Tel Aviv, ISRAEL.

E-mail address: aaro@tau.ac.il

[Lemańczyk] Faculty of Mathematics and Computer Science, Nicolaus Copernicus University, ul. Chopina 12/18, 87-100 Toruń, Poland

E-mail address: mlem@mat.uni.torun.pl 